TRANSACTIONS OF THE

AMERICAN MATHEMATICAL SOCIETY

Volume 355, Number 9, Pages 3777-3794

S 0002-9947(03)03299-9

Article electronically published on May 29, 2003

\title{
ASYMPTOTICS FOR LOGICAL LIMIT LAWS: WHEN THE GROWTH OF THE COMPONENTS IS IN AN RT CLASS
}

\author{
JASON P. BELL AND STANLEY N. BURRIS
}

\begin{abstract}
Compton's method of proving monadic second-order limit laws is based on analyzing the generating function of a class of finite structures. For applications of his deeper results we previously relied on asymptotics obtained using Cauchy's integral formula. In this paper we develop elementary techniques, based on a Tauberian theorem of Schur, that significantly extend the classes of structures for which we know that Compton's theory can be applied.
\end{abstract}

\section{INTRODUCTION}

We are primarily interested in being able to show that an answer exists to the following question: given a class $\mathcal{K}$ of finite relational structures and a property $\Phi$ :

What is the probability that a finite structure, randomly selected

from $\mathcal{K}$, has the property $\Phi$ ?

While pursuing this goal we are able to prove (in Corollary 4.3) the conjecture ([10], p. 462) of Durrett, Granovsky and Gueron arising in the study of coagulationfragmentation processes which says that if $\mathbf{S}(x)=\sum s(n) x^{n}$ is a power series with positive coefficients such that $s(n-1) / s(n) \rightarrow \rho$, then the coefficients of the power series expansion of $\exp (\mathbf{S}(x))$ have the same property.

The notion of probability that we use is to take the proportion $q_{n}$ of finite structures of size $n$ in $\mathcal{K}$ (we only count up to isomorphism) that have the property $\Phi$, and then to take the limit $q$ of the sequence $q_{n}$ as $n$ goes to infinity. This limit, when it exists, is also called the asymptotic density of the class of members of $\mathcal{K}$ that satisfy $\Phi$.

It has been known since the mid 1970s that a few well-known classes, like graphs or directed graphs, are such that if $\Phi$ is a property defined by a first-order sentence, then the probability must exist, and be 0 or 1 . If $\mathcal{K}$ is a class such that every sentence in a given (logic) language $L$ defines a property for which a probability exists, then we say $\mathcal{K}$ has an L-limit law.

In the 1980s Kevin Compton gave a new method for proving that a class $\mathcal{K}$ of relational structures has a monadic second-order limit law, a method that only depends on analyzing the growth rate of $a(n)$, the number of structures of size $n$ in $\mathcal{K}$. This applies to classes that are closed under disjoint union and components.

Received by the editors June 26, 2002 and, in revised form, January 10, 2003.

2000 Mathematics Subject Classification. Primary 03C13, 05A16, 11P99, 41A60; Secondary 11N45, 11N80, 11U99, 60J20.

Key words and phrases. Ratio test, Schur's Tauberian theorem, asymptotic density, monadic second-order logic, zero-one law, limit law.

The second author would like to thank NSERC for support of this research.

(C)2003 American Mathematical Society 
For such classes the count function $p(n)$, the number of components of size $n$ in $\mathcal{K}$, is often more readily available than $a(n)$, and we would like to know conditions on $p(n)$ that guarantee a logical limit law. The best results of this type previously known were

- if $p(n)=\mathrm{O}\left(n^{c}\right)$, that is, $p(n)$ is polynomially bounded, then $\mathcal{K}$ has a monadic second-order 0-1 law (Bell [1]), and

- if $p(n)=C \beta^{n}+\mathrm{O}\left(\gamma^{n}\right)$, where $0<\gamma<\beta$ and $C>0$, then $\mathcal{K}$ has a monadic second-order limit law (see [4], Chapters 5 and 6).

We will make use of a Tauberian theorem of Schur to extend these results to cover a large number of new classes of structures. For example, we will show that

$$
p(n) \sim a n^{b} e^{c n^{d}}
$$

with $d<1$ and $a, c>0$, yields a monadic second-order 0-1 law; and

Corollary 9.4. For $\mu<1<\beta$ and $C>0$, or $\mu=1<\beta$ and $C>1$, the condition

$$
p(n) \sim C \beta^{n} / n^{\mu}
$$

guarantees that $\mathcal{K}$ has a monadic second-order limit law.

In addition to the standard "big O" and "little o" notation from asymptotics we use the following:

\begin{tabular}{ll} 
notation & means \\
\hline$f(n) \prec g(n)$ & $f(n)$ is eventually less than $g(n)$ \\
$f(n) \preccurlyeq g(n)$ & $f(n)$ is eventually less than or equal to $g(n)$ \\
$f(n) \succ g(n)$ & $f(n)$ is eventually greater than $g(n)$ \\
$f(n) \succcurlyeq g(n)$ & $f(n)$ is eventually greater than or equal to $g(n)$
\end{tabular}

As mathematics symbols we will be using upper case boldface roman letters exclusively to denote power series, and the corresponding lower case ordinary italic letters name the coefficients. Thus we will use $\mathbf{A}(x)=\sum_{n} a(n) x^{n}, \ldots, \mathbf{T}(x)=$ $\sum_{n} t(n) x^{n}$.

\section{THE CLASS RT $\rho$}

The sequences in $\mathrm{RT}_{\rho}$ play a central role in the study of Compton's development of logical limit laws. We find conditions to guarantee membership in this class and then apply them to prove logical limit laws.

Definition 2.1. $\mathrm{RT}_{\rho}$ is the collection of sequences $s(n)$ of real numbers that satisfy

(a) $s(n) \succ 0$, and

(b) $\lim _{n \rightarrow \infty} \frac{s(n-1)}{s(n)}=\rho$.

We also say that a power series $\mathbf{S}(x)$ is in $\mathrm{RT}_{\rho}$ if its sequence of coefficients $s(n)$ is in $\mathrm{RT}_{\rho}$. Also, if $f(x)$ is a function that admits a power series expansion $\sum s(n) x^{n}$ about 0 , then we say $f(x)$ is in $\mathrm{RT}_{\rho}$ if $s(n)$ is in $\mathrm{RT}_{\rho}$.

The following lemma and corollary give the most basic information about the growth rate of members of $\mathrm{RT}_{\rho}$.

Lemma 2.2. If $s(n) \in \mathrm{RT}_{\rho}$ with $0<\rho<\infty$ then, for $0<\varepsilon<\rho^{-1}$, there is an $N$ such that, for $n \geq N$,

$$
\left(\rho^{-1}-\varepsilon\right)^{n}<s(n)<\left(\rho^{-1}+\varepsilon\right)^{n} .
$$


From this we see that a smaller $\rho$ leads to much faster-growing sequences.

Corollary 2.3. If $s(n) \in \mathrm{RT}_{\sigma}$ and $t(n) \in \mathrm{RT}_{\tau}$ with $0<\sigma<\tau<\infty$, then $t(n)=\mathrm{o}(s(n))$.

The class $\mathrm{RT}_{\rho}$ has some remarkable similarities to the class $\mathrm{RV}_{\alpha}$, the class of functions of regular variation at infinity with index $\alpha$, but this connection does not seem to have been thoroughly researched. For $0<\rho<\infty$ the sequence $\rho^{-n}$ is perhaps the simplest member of the class $\mathrm{RT}_{\rho}$, and this sequence, along with $\mathrm{RT}_{1}$, completely determines $\mathrm{RT}_{\rho}$, since one can easily check that

$$
s(n) \in \mathrm{RT}_{\rho} \Longleftrightarrow s(n) \rho^{n} \in \mathrm{RT}_{1} .
$$

In terms of power series this would be written as

$$
\mathbf{S}(x) \in \mathrm{RT}_{\rho} \Longleftrightarrow \mathrm{S}(\rho x) \in \mathrm{RT}_{1} .
$$

Here are three of the simplest examples from $\mathrm{RT}_{1}$ :

$$
\begin{array}{ll}
s(n)=c & \text { for } c>0, \\
s(n)=n^{c} & \text { for } c \text { any real number, } \\
s(n)=d^{n^{c}} & \text { for } c<1<d .
\end{array}
$$

We can use these examples, with Proposition 2.4, to make a substantial collection of sequences in $\mathrm{RT}_{\rho}$. Multiplication of the sequences $s(n)$ and $t(n)$ gives $s(n) \cdot t(n)$, and division gives $s(n) / t(n)$, where we define $s(n) / t(n)$ to be 0 whenever $t(n)=0$.

Proposition 2.4. $\mathrm{RT}_{1}$ is closed under multiplication, division, and asymptotically equal. For $0<\sigma, \tau<\infty$, if $s(n) \in \mathrm{RT}_{\sigma}$ and $t(n) \in \mathrm{RT}_{\tau}$, then $1 / s(n) \in \mathrm{RT}_{1 / \sigma}$, and $s(n) \cdot t(n) \in \mathrm{RT}_{\sigma \tau}$. Furthermore, $\mathbf{S}(x) \in \mathrm{RT}_{\rho}$ iff $\mathbf{S}^{\prime}(x) \in \mathrm{RT}_{\rho}$ iff $x \mathbf{S}(x) \in \mathrm{RT}_{\rho}$.

Proof. (Straightforward)

With this we can easily see, for example, that for $a, c, \rho>0$ and $b$ any real number, if $s(n) \sim a n^{b} e^{c \sqrt{n}} / \rho^{n}$, then $s(n) \in \mathrm{RT}_{\rho}$. Just such an example played an important role in finding the first applications of Compton's 1989 theoretical development of logical limit laws. A key fact about this particular sequence is that $a n^{b} e^{c \sqrt{n}} \in \mathrm{RT}_{1}$, and it is eventually nondecreasing.

\section{The Cauchy product}

The Cauchy product $\mathbf{R}(x)=\mathbf{S}(x) \cdot \mathbf{T}(x)$ is defined by

$$
r(n)=\sum_{k=0}^{n} s(k) \cdot t(n-k) .
$$

The following two lemmas and corollary help us extract information about RT $\rho$ classes from the Cauchy product. We start with the classic Tauberian theorem of Schur 1

Lemma 3.1 (Schur). With $0 \leq \rho<\infty$, suppose that

(a) $\mathbf{A}(x) \in \mathrm{RT}_{\rho}$,

\footnotetext{
${ }^{1}$ It is a Tauberian theorem since it gives an extra condition, namely the radius of convergence of $\mathbf{B}(x)>\rho$, to allow us to go from a generalized limit, $\lim _{x \rightarrow \rho} \mathbf{C}(x) / \mathbf{A}(x)$, to an ordinary limit, $\lim _{n \rightarrow \infty} c(n) / a(n)$.
} 
(b) $\mathbf{B}(x)$ has radius of convergence greater than $\rho$, and

(c) $\mathbf{B}(\rho)>0$.

Let $\mathbf{C}(x)=\mathbf{A}(x) \cdot \mathbf{B}(x)$. Then

$$
c(n) \sim \mathbf{B}(\rho) \cdot a(n) .
$$

Proof. (See Bender [3] or Burris [4.)

Thus from the hypotheses of Schur's Lemma we deduce that $\mathbf{C}(x) \in \mathrm{RT}_{\rho}$.

Corollary 3.2. With $0<\rho<\infty$, suppose $\mathbf{A}(x) \in \mathrm{RT}_{\rho}$ and the radius of convergence of $\mathbf{B}(x)$ is greater than $\rho$. If $\mathbf{B}(\rho)>0$, then

$$
\mathbf{A}(x) \cdot \mathbf{B}(x) \in \mathrm{RT}_{\rho} .
$$

Proof. Let $\mathbf{C}(x)=\mathbf{A}(x) \cdot \mathbf{B}(x)$. From Schur's Lemma we have

$$
c(n) \sim \mathbf{B}(\rho) \cdot a(n)
$$

so, by Proposition 2.4, $\mathbf{C}(x) \in \mathrm{RT}_{\rho}$.

The next lemma offers a variation on this theme.

Lemma 3.3. Suppose $\mathbf{C}(x)=\mathbf{A}(x) \cdot \mathbf{B}(x)$, where $\mathbf{A}(x)$ and $\mathbf{B}(x)$ have nonnegative coefficients and $\mathbf{B}(x)$ is not the zero power series. If

(a) $\mathbf{A}(x) \in \mathrm{RT}_{\rho}$, where $0<\rho<\infty$,

(b) $b(n)=\mathrm{o}(c(n))$, and

(c) $\frac{c(n-1)}{c(n)} \preccurlyeq \rho$,

then $\mathbf{C}(x) \in \mathrm{RT}_{\rho}$.

Proof. From the following equivalent statements,

$$
\begin{array}{lll}
\mathbf{A}(x)=\mathbf{B}(x) \cdot \mathbf{C}(x) & \text { iff } & \mathbf{A}(\rho x)=\mathbf{B}(\rho x) \cdot \mathbf{C}(\rho x), \\
\mathbf{A}(x) \in \mathrm{RT}_{\rho} & \text { iff } & \mathbf{A}(\rho x) \in \mathrm{RT}_{1}, \\
b(n)=\mathrm{o}(c(n)) & \text { iff } & b(n) \rho^{n}=\mathrm{o}\left(c(n) \rho^{n}\right), \\
\frac{c(n-1)}{c(n)} \preccurlyeq \rho & \text { iff } & \frac{c(n-1) \rho^{n-1}}{c(n) \rho^{n}} \preccurlyeq 1, \\
\mathbf{C}(x) \in \mathrm{RT}_{\rho} & \text { iff } & \mathbf{C}(\rho x) \in \mathrm{RT}_{1},
\end{array}
$$

it suffices to prove the lemma in the case that $\rho=1$. With $\rho=1$ the goal is:

$$
\text { from }\left(\mathrm{a}^{\prime}\right) \mathbf{A}(x) \in \mathrm{RT}_{1},\left(\mathrm{~b}^{\prime}\right) b(n)=\mathrm{o}(c(n)) \text {, and }\left(\mathrm{c}^{\prime}\right) c(n-1) \preccurlyeq c(n) \text {, }
$$
prove that $\mathbf{C}(x) \in \mathrm{RT}_{1}$.

Let $\varepsilon>0$. By $\left(\mathrm{a}^{\prime}\right)$ there exists an integer $M$ such that, for $n>M$,

$$
|a(n)-a(n-1)|<\varepsilon a(n) .
$$

Since $\mathbf{A}(x) \in \mathrm{RT}_{1}$ implies $a(n) \succ 0$, and $\mathbf{B}(x)$ is not the zero power series, we have $c(n) \succ 0$. So, in view of $\left(\mathrm{c}^{\prime}\right)$ we can also assume that $M$ is sufficiently large to guarantee

$$
n \geq M \Longrightarrow\left\{\begin{array}{l}
c(n)>0, \quad \text { and } \\
\frac{c(n-1)}{c(n)} \leq 1 .
\end{array}\right.
$$

By assumption $\left(\mathrm{b}^{\prime}\right)$ we can find an integer $N>M$ such that 


$$
b(n) \leq \frac{\varepsilon}{(M+1) \max (1, a(0), \ldots, a(M))} \cdot c(n)
$$

for $n \geq N-M$.

Now suppose $n \geq M+N$. Then, by (3.2),

$$
0<c(n-M) \leq \cdots \leq c(n-1) \leq c(n) .
$$

For $n \geq M+N$,

$$
\begin{aligned}
c(n)-c(n-1)= & \sum_{i=0}^{n} a(n-i) \cdot b(i)-\sum_{i=0}^{n-1} a(n-1-i) \cdot b(i) \\
= & \sum_{i<n-M}(a(n-i)-a(n-1-i)) \cdot b(i) \\
& \quad+\sum_{i=n-M}^{n} a(n-i) \cdot b(i)-\sum_{i=n-M}^{n-1} a(n-1-i) \cdot b(i) \\
\leq & \varepsilon \sum_{i=0}^{n} a(n-i) \cdot b(i)+\sum_{i=n-M}^{n} \varepsilon c(i) /(M+1) \quad \text { by (3.1) , (3.3) } \\
\leq & \varepsilon c(n)+\sum_{i=n-M}^{n} \varepsilon c(n) /(M+1) \text { by (3.4) } \\
= & 2 \varepsilon c(n) .
\end{aligned}
$$

Therefore, $1-2 \varepsilon \leq \frac{c(n-1)}{c(n)} \leq 1$. Since this holds for any $n>M+N$, we have $\lim _{n \rightarrow \infty} \frac{c(n-1)}{c(n)}=1$, and so $\mathbf{C}(x) \in \mathrm{RT}_{1}$.

\section{EXPONENTIATION}

We will be particularly interested in knowing that exponentiation of a power series preserves membership in $\mathrm{RT}_{\rho}$. First we prove a special case of this result.

Lemma 4.1. Let $\mathbf{T}(x)=\exp (\mathbf{S}(x))$, where

(a) $\mathbf{S}(x) \in \mathrm{RT}_{1}$,

(b) the $s(n)$ are nonnegative, and

(c) $\liminf _{n \rightarrow \infty} \frac{t(n)}{t(n-1)}=C>0$.

Then $\mathbf{T}(x) \in \mathrm{RT}_{1}$.

Proof. Note that $C \leq 1$ since the radius of convergence of $\mathbf{T}(x)$ is 1 . From $\mathbf{S}(x) \in$ $\mathrm{RT}_{1}$ we know that $x \mathbf{S}^{\prime}(x) \in \mathrm{RT}_{1}$. So, given $\varepsilon>0$, we can choose $M$ such that

$$
|m s(m)-(m-1) s(m-1)|<\varepsilon m s(m)
$$

for $m \geq M$. Also, we can choose $N$ such that $\frac{t(n)}{t(n-1)}>\frac{C}{2}$ for $n>N$. From this we see that

$$
n-r>N \Longrightarrow \frac{t(n)}{t(n-r)}>\frac{C^{r}}{2^{r}}
$$


Differentiating $\mathbf{T}(x)=\exp (\mathbf{S}(x))$ we have

$$
\mathbf{T}^{\prime}(x)=\mathbf{S}^{\prime}(x) \mathbf{T}(x),
$$

and equating the coefficients of $x^{n-1}$ on both sides of this equation gives

$$
n t(n)=\sum_{m \leq n} t(m) \cdot(n-m) s(n-m) .
$$

From this it follows that if $n>N+M$ (we adopt the convention that $s(m)=0$ for $m<0)$,

$$
\begin{aligned}
\mid n t(n) & -(n-1) t(n-1) \mid \\
= & \left|\sum_{m \leq n} t(m) \cdot((n-m) s(n-m)-(n-1-m) s(n-1-m))\right| \\
\leq & \sum_{0 \leq m \leq n-M} t(m) \cdot \varepsilon(n-m) s(n-m) \\
& \quad+\sum_{n-M<m \leq n} t(m) \cdot|(n-m) s(n-m)-(n-1-m) s(n-1-m)| \\
\leq \quad & \operatorname{\varepsilon nt}(n)+2 \cdot\left(\max _{0 \leq m \leq M} m s(m)\right) \cdot \sum_{n-M<m \leq n} t(m) \quad \text { by (4.2) } \\
\leq \quad & \operatorname{\varepsilon nt}(n)+2 M \cdot\left(\max _{0 \leq m \leq M} s(m)\right) \cdot M 2^{M} \cdot C^{-M} \cdot t(n) \quad \text { by (4.1) } \\
= & (\varepsilon+o(1)) n t(n) .
\end{aligned}
$$

Thus $n t(n) \in \mathrm{RT}_{1}$; so $t(n) \in \mathrm{RT}_{1}$.

Lemma 4.2. $\quad \mathbf{S}(x) \in \mathrm{RT}_{1} \Longrightarrow \exp (\mathbf{S}(x)) \in \mathrm{RT}_{1}$.

Proof. Let $\mathbf{T}(x)=\exp (\mathbf{S}(x))$. Since $s(n) \in \mathrm{RT}_{1}$ and $2^{-n} \in \mathrm{RT}_{2}$, by Corollary 2.3 there exists $N$ such that $s(n)>2^{-n}$ for $n>N$. Define $\widehat{s}(n)$ to be 1 for $0 \leq n \leq N$ and to be $s(n)$ for $n>N$. Let $\widehat{\mathbf{S}}(x)=\sum_{n} \widehat{s}(n) x^{n}$, and let $\widehat{\mathbf{T}}(x)=\sum_{n} \widehat{t}(n) x^{n}$ be such that $\widehat{\mathbf{T}}(x)=\exp (\widehat{\mathbf{S}}(x))$. Since

$$
\widehat{s}(n) \geq 2^{-n} / n, \quad \text { for } n \geq 1,
$$

$\widehat{\mathbf{S}}(x)+\log (1-x / 2)$ is a power series with nonnegative coefficients. Thus, for $n \geq 0$,

$$
\begin{aligned}
\widehat{t}(n)-\widehat{t}(n-1) / 2 & =\left[x^{n}\right](1-x / 2) \widehat{\mathbf{T}}(x) \\
& =\left[x^{n}\right] \exp (\log (1-x / 2)+\widehat{\mathbf{S}}(x)) \\
& \geq 0
\end{aligned}
$$

so

$$
\liminf _{n \rightarrow \infty} \frac{\widehat{t}(n)}{\widehat{t}(n-1)} \geq 1 / 2>0 .
$$

Now $\widehat{\mathbf{T}}(x) \in \mathrm{RT}_{1}$ by Lemma 4.1 since $\widehat{\mathbf{S}}(x) \in \mathrm{RT}_{1}$. To finish the proof, observe that

where $\mathbf{p}(x)$ is a polynomial; so

$$
\mathbf{S}(x)-\widehat{\mathbf{S}}(x)=\mathbf{p}(x),
$$

$$
\mathbf{T}(x)=\exp (\mathbf{p}(x)) \cdot \widehat{\mathbf{T}}(x)
$$

By Corollary 3.2, $\mathbf{T}(x) \in \mathrm{RT}_{1}$. 
Next we see that $\mathrm{RT}_{\rho}$ classes are closed under exponentiation of power series, proving the conjecture ([10], p. 462) of Durrett, Granovsky and Gueron.

Corollary 4.3. For $0<\rho<\infty$,

$$
\mathbf{S}(x) \in \mathrm{RT}_{\rho} \quad \Longrightarrow \quad \exp (\mathbf{S}(x)) \in \mathrm{RT}_{\rho} .
$$

Proof. This follows from (2.1) and Lemma 4.2.

If we exponentiate a power series in $\mathrm{RT}_{\rho}$ that diverges at $\rho$, then we obtain a power series whose coefficients grow much faster than those of the original series.

Lemma 4.4. Let $\mathbf{T}(x)=\exp (\mathbf{S}(x))$ where

(a) $\mathbf{S}(x) \in \mathrm{RT}_{\rho} \quad$ with $0<\rho<\infty$, and

(b) $\mathbf{S}(\rho)=\infty$.

Then $s(n)=\mathrm{o}(t(n))$.

Proof. First we prove this lemma for the case that the coefficients of $\mathbf{S}(x)$ are nonnegative. Choose $N \geq 1$ and observe that, for $n \geq N$,

$$
t(n)=\sum_{j \geq 0} \frac{1}{j !}\left[x^{n}\right] \mathbf{S}(x)^{j} \geq \frac{1}{2}\left[x^{n}\right] \mathbf{S}(x)^{2} \geq \frac{1}{2} \sum_{k=0}^{N} s(k) s(n-k)
$$

and thus

$$
\frac{t(n)}{s(n)} \geq \frac{1}{2} \sum_{k=0}^{N} s(k) \frac{s(n-k)}{s(n)} .
$$

Taking the $\liminf _{n \rightarrow \infty}$ of both sides, using the fact that $\mathbf{S}(x) \in \mathrm{RT}_{\rho}$, gives

$$
\liminf _{n \rightarrow \infty} \frac{t(n)}{s(n)} \geq \frac{1}{2} \sum_{k=0}^{N} s(k) \rho^{k} .
$$

Now use the fact that $\mathbf{S}(\rho)=\infty$.

For the general case, where some of the coefficients of $\mathbf{S}(x)$ can be negative, let $\mathbf{p}(x)$ be a polynomial such that $\widehat{\mathbf{S}}(x)=\mathbf{S}(x)+\mathbf{p}(x)$ has nonnegative coefficients. Clearly $\widehat{\mathbf{S}}(x) \in \mathrm{RT}_{\rho}$ and $\widehat{\mathbf{S}}(\rho)=\infty$. Then $\widehat{\mathbf{T}}(x)=\exp (\widehat{\mathbf{S}}(x))$ is such that

$$
\widehat{s}(n)=\mathrm{o}(\widehat{t}(n)),
$$

by the first part of the proof. By Corollary $4.3 \widehat{\mathbf{T}}(x) \in \mathrm{RT}_{\rho}$. Since

$$
\mathbf{T}(x)=\exp (-\mathbf{p}(x)) \cdot \widehat{\mathbf{T}}(x),
$$

by Schur's Lemma we have

$$
t(n) \sim \exp (-\mathbf{p}(\rho)) \cdot \widehat{t}(n) .
$$

So from (4.3) we have $s(n)=\mathrm{o}(t(n))$ since $s(n)$ eventually equals $\widehat{s}(n)$.

A modest growth condition on the coefficients of $\mathbf{S}(x)$ guarantees that the coefficients of $\exp (\mathbf{S}(x))$ satisfy a growth condition used to prove logical limit laws. But first we prove a technical lemma on membership in $\mathrm{RT}_{1}$.

Lemma 4.5. Suppose $\mathbf{S}(x) \in \mathrm{RT}_{1}$. Then

$$
\liminf _{n \rightarrow \infty} s(n)>1 \Longrightarrow s(n)-1 \in \mathrm{RT}_{1} .
$$


Proof. Choose $C, N>1$ such that $s(n) \geq C$, for $n \geq N$. Then, for $n \geq N$,

$$
1-\frac{1}{s(n)} \geq \frac{C-1}{s(n)} \geq C-1>0 \text {. }
$$

Now

$$
\frac{s(n-1)-1}{s(n)-1}-1=\frac{\frac{s(n-1)}{s(n)}-1}{1-\frac{1}{s(n)}},
$$

and the right side tends to 0 as $n$ tends to infinity since $s(n) \in \mathrm{RT}_{1}$ says the numerator tends to 0 , and (4.4) says the denominator is bounded away from 0 . Thus,

$$
\lim _{n \rightarrow \infty} \frac{s(n-1)-1}{s(n)-1}=1
$$

Now we proceed with the analysis of the growth rate of the coefficients after exponentiation.

Lemma 4.6. Let $\mathbf{T}(x)=\exp (\mathbf{S}(x))$ where

(a) $\mathbf{S}(x) \in \mathrm{RT}_{\rho}$ with $0<\rho<\infty$, and

(b) $\liminf _{n \rightarrow \infty} n s(n) \rho^{n}>1$.

Then $\mathbf{T}(x) \in \mathrm{RT}_{\rho}$ and $\frac{t(n-1)}{t(n)} \prec \rho$.

Proof. Corollary 4.3 gives $\mathbf{T}(x) \in \mathrm{RT}_{\rho}$. To verify $t(n-1) \prec t(n) \rho$ note that

$$
\begin{aligned}
t(n) \rho^{n}-t(n-1) \rho^{n-1} & =\left[x^{n}\right]((1-x) \cdot \mathbf{T}(\rho x)) \\
& =\left[x^{n}\right]((1-x) \cdot \exp (\mathbf{S}(\rho x))) \\
& =\left[x^{n}\right] \exp \left(s(0)+\sum_{n \geq 1}\left(s(n) \rho^{n}-1 / n\right) x^{n}\right) .
\end{aligned}
$$

Since $\mathbf{S}(x) \in \mathrm{RT}_{\rho}$,

$$
n s(n) \rho^{n} \in \mathrm{RT}_{1},
$$

and then Lemma 4.5 gives $n s(n) \rho^{n}-1 \in \mathrm{RT}_{1}$. Using Proposition 2.4 we have

$$
s(n) \rho^{n}-1 / n \in \mathrm{RT}_{1} \text {. }
$$

Choose $N \geq 1$ such that, for $n \geq N$,

$$
n s(n) \rho^{n}>1,
$$

and let

$$
\begin{aligned}
\mathbf{p}(x) & =s(0)+\sum_{n=1}^{N-1}\left(s(n) \rho^{n}-1 / n\right) x^{n}-\sum_{n=1}^{N-1} x^{n}, \\
\mathbf{R}(x) & =\sum_{n=1}^{N-1} x^{n}+\sum_{n=N}^{\infty}\left(s(n) \rho^{n}-1 / n\right) x^{n} .
\end{aligned}
$$

By (4.5) we know that $\mathbf{R}(x) \in \mathrm{RT}_{1}$; so Lemma 4.2 gives

$$
\exp (\mathbf{R}(x)) \in \mathbf{R T}_{1} \text {. }
$$


Noting that $\mathbf{p}(x)$ is a polynomial we have

$$
\begin{aligned}
t(n) \rho^{n}-t(n-1) \rho^{n-1} & =\left[x^{n}\right] \exp \left(s(0)+\sum_{n \geq 1}\left(s(n) \rho^{n}-1 / n\right)\right. \\
& =\left[x^{n}\right](\exp (\mathbf{p}(x)) \cdot \exp (\mathbf{R}(x))) \\
& \sim \exp (\mathbf{p}(1)) \cdot\left[x^{n}\right] \exp (\mathbf{R}(x))
\end{aligned}
$$

by (4.7) and Schur's Lemma, and thus, since the coefficients of $\mathbf{R}(x)$ are positive,

$$
t(n) \rho^{n}-t(n-1) \rho^{n-1} \succ 0 .
$$

This says $\frac{t(n-1)}{t(n)} \prec \rho$.

\section{THE STAR TRANSFORMATiON}

Now we introduce a transformation on a power series $\mathbf{S}(x)$ that plays an important role in combinatorics.

Definition 5.1. Let $\mathbf{S}^{\star}(x)=\sum s^{\star}(n) x^{n}$ be the power series defined by

$$
\begin{aligned}
& s^{\star}(0)=0, \\
& s^{\star}(n)=\sum_{j k=n} s(j) / k \quad \text { for } n \geq 1 .
\end{aligned}
$$

Lemma 5.2. For $0<\rho<1$, if $\mathbf{S}(x) \in \mathrm{RT}_{\rho}$ has nonnegative coefficients, then

(a) $s^{\star}(n) \sim s(n)$, and

(b) $\mathbf{S}^{\star}(x) \in \mathrm{RT}_{\rho}$.

Proof. Since $s(n) \in \mathrm{RT}_{\rho}$ we know from Corollary 2.3 that $0<\beta<\rho^{-1}<\alpha$ implies

$$
n^{2} \beta^{n}=\mathrm{o}(s(n)) \text { and } s(n)=\mathrm{o}\left(\alpha^{n}\right)
$$

since $n^{2} \beta^{n} \in \mathrm{RT}_{1 / \beta}$ and $\alpha^{n} \in \mathrm{RT}_{1 / \alpha}$. Choose $\alpha$ satisfying $\rho^{-1}<\alpha<\rho^{-2}$ and choose $C$ such that $|s(n)|<C \alpha^{n}$ for all $n$. Choose $N$ such that $s(n) \geq 0$ for $n \geq N$. Then

$$
\begin{aligned}
n s^{\star}(n) & =\sum_{d \mid n} d s(d) \\
& \leq n s(n)+\sum_{d \leq n / 2} d s(d) \\
& \leq n s(n)+\sum_{d \leq n / 2} d C \alpha^{n / 2} \\
& =n s(n)+\mathrm{O}\left(n^{2} \alpha^{n / 2}\right) \\
& =n s(n)+\mathrm{O}(s(n)) \quad\left(\text { since } \sqrt{\alpha}<\rho^{-1}\right) .
\end{aligned}
$$

Hence $n s^{\star}(n) \sim n s(n)$ since $0 \leq s(n) \leq s^{\star}(n)$, and thus $s^{\star}(n) \sim s(n)$, giving (a). Then from Proposition 2.4 we have $\mathbf{S}^{\star}(x) \in \mathrm{RT}_{\rho}$.

This result is best possible since one can easily find examples with $\rho=1$ such that $\mathbf{S}(x) \in \mathrm{RT}_{1}$ but $\mathbf{S}^{\star}(x) \notin \mathrm{RT}_{1}$. For example, $\mathbf{S}(x)=\sum_{n} x^{n} \in \mathrm{RT}_{1}$, but $\mathbf{S}^{\star}(x)=\sum_{n} \sigma(n) x^{n} / n \notin \mathrm{RT}_{1}$. However, the power series expansion of $\frac{x}{1-x} \cdot \mathbf{S}^{\star \prime}(x)$ is much better behaved in this situation. 
Lemma 5.3. Suppose $s(n) \in \mathrm{RT}_{1}$ is a sequence of nonnegative terms. Then

$$
s^{\star}(1)+\cdots+n s^{\star}(n) \in \mathrm{RT}_{1} .
$$

Proof. Let $S^{\star}(n)=s^{\star}(1)+\cdots+n s^{\star}(n)$. Then

$$
S^{\star}(n)=\sum_{j=1}^{n}\left\lfloor\frac{n}{j}\right\rfloor j s(j)
$$

since

$$
S^{\star}(n)=\sum_{m=1}^{n} m s^{\star}(m)=\sum_{j k=1}^{n} j k \cdot s(j) / k=\sum_{j k=1}^{n} j s(j)=\sum_{j=1}^{n}\left\lfloor\frac{n}{j}\right\rfloor j s(j) .
$$

Also,

$$
S^{\star}(n)-S^{\star}(n-1)=n s^{\star}(n)=\sum_{d \mid n} d s(d) .
$$

We shall show that $S^{\star}(n)-S^{\star}(n-1)$ is o $\left(S^{\star}(n)\right)$.

Fix $\varepsilon \in(0,1)$ and choose

$$
M>\frac{1}{\varepsilon(1-\varepsilon)} .
$$

For any fixed integer $r, \frac{(n-r) s(n-r)}{n s(n)} \rightarrow 1$ as $n$ tends to infinity. Hence we can choose $N>M^{3}$ such that

$$
|n s(n)-(n-r) s(n-r)|<\varepsilon n s(n)
$$

for $0 \leq r \leq M$ and $n \geq N / M$, and thus

$$
(n-r) s(n-r)>(1-\varepsilon) n s(n)
$$

for $0 \leq r \leq M$ and $n \geq N / M$.

For integers $d_{1}, d_{2}$ with $1 \leq d_{1}<d_{2} \leq M$, and for $n \geq N$, we have

$$
\frac{n}{d_{1}}-\frac{n}{d_{2}}=\frac{n\left(d_{2}-d_{1}\right)}{d_{1} d_{2}} \geq \frac{n}{M^{2}}>\frac{M^{3}}{M^{2}}=M,
$$

and thus $\frac{n}{d_{2}}<\frac{n}{d_{1}}-M$.

Consequently, for $n \geq N$, if $d_{1}<\cdots<d_{k}$ are the divisors of $n$ from the interval $[1, M]$ we see that

$$
\frac{n}{M}<\frac{n}{d_{k}}-M<\frac{n}{d_{k}}<\frac{n}{d_{k-1}}-M<\cdots<\frac{n}{d_{1}}-M<\frac{n}{d_{1}}
$$


Returning to the expression for $S^{\star}(n)$ in (5.1), now assuming that $n \geq N$, we have

$$
\begin{aligned}
S^{\star}(n) & =\sum_{j=1}^{n}\left\lfloor\frac{n}{j}\right\rfloor j s(j) \\
& =\sum_{1 \leq j \leq n / M}\left\lfloor\frac{n}{j}\right\rfloor j s(j)+\sum_{n / M<j \leq n}\left\lfloor\frac{n}{j}\right\rfloor j s(j) \\
& \geq \sum_{1 \leq j \leq n / M} M j s(j)+\sum_{n / M<j \leq n} j s(j) \\
& \geq \frac{1}{\varepsilon} \sum_{1 \leq j \leq n / M} j s(j)+\sum_{\substack{d \mid n \\
d<M}}\left(\sum_{\substack{n / d-M \\
j \leq n / d}} j s(j)\right) \quad \text { by (5.3) },(5.5) \\
& \geq \frac{1}{\varepsilon} \sum_{\substack{d \mid n \\
d \leq n / M}} d s(d)+\sum_{\substack{d \mid n \\
d<M}} M(1-\varepsilon) \frac{n}{d} \cdot s\left(\frac{n}{d}\right) \quad \text { by (15.4) } \\
& \geq \frac{1}{\varepsilon} \sum_{\substack{d \mid n \\
d \leq n / M}} d s(d)+\frac{1}{\varepsilon} \sum_{\substack{d \mid n \\
d>n / M}} d s(d) \text { by (5.3) } \\
& =\frac{1}{\varepsilon} \sum_{d \mid n} d s(d) \\
& =\frac{1}{\varepsilon}\left(S^{\star}(n)-S^{\star}(n-1)\right) \quad \text { by (5.2). }
\end{aligned}
$$

Thus $0 \leq S^{\star}(n)-S^{\star}(n-1) \leq \varepsilon S^{\star}(n)$, and so $S^{\star}(n) \in \mathrm{RT}_{1}$.

\section{Combining Star With exponentiation}

Theorem 6.1. Let $\mathbf{T}(x)=\exp \left(\mathbf{S}^{\star}(x)\right)$, where

(a) $\mathbf{S}(x) \in \mathbf{R T}_{\rho}$ with $0<\rho<1$,

(b) the $s(n)$ are nonnegative, and

(c) $\liminf _{n \rightarrow \infty} n s(n) \rho^{n}>1$.

Then $\mathbf{T}(x) \in \mathrm{RT}_{\rho}$ and $\frac{t(n-1)}{t(n)} \prec \rho$.

Furthermore, if $\mathbf{S}(\rho)=\infty$, then $s(n)=\mathrm{o}(t(n))$.

Proof. $\mathbf{S}^{\star}(x) \in \mathrm{RT}_{\rho}$ by Lemma [5.2, so $\mathbf{T}(x) \in \mathrm{RT}_{\rho}$ by Corollary 4.3 . From $0 \leq$ $s(n) \leq s^{\star}(n)$ we have $\liminf _{n \rightarrow \infty} n s^{\star}(n) \rho^{n}>1$. Thus $t(n-1) \prec t(n) \rho$ by Lemma 4.6

For the final assertion assume $\mathbf{S}(\rho)=\infty$. Then $\mathbf{S}^{\star}(\rho)=\infty$; so from Lemma 4.4 we have $s^{\star}(n)=\mathrm{o}(t(n))$, and thus $s(n)=\mathrm{o}(t(n))$.

When $\rho=1$ we can no longer assume $\mathbf{S}^{\star}(x) \in \mathrm{RT}_{1}$ just because $\mathbf{S}(x) \in \mathrm{RT}_{1}$. Instead we turn to $x \mathbf{S}^{\star^{\prime}}(x) /(1-x)$ to find a well-behaved sequence of coefficients.

Theorem 6.2. Let $\mathbf{T}(x)=\exp \left(\mathbf{S}^{\star}(x)\right)$ where

(a) $\mathbf{S}(x) \in \mathrm{RT}_{1}$,

(b) the $s(n)$ are nonnegative, and

(c) $s(n) \succcurlyeq 1 / n$.

Then $\exp \left(\mathbf{S}^{\star}(x)\right) \in \mathrm{RT}_{1}$. Furthermore, $s(n)=\mathrm{o}(t(n))$. 
Proof. From

$$
s^{\star}(n) \geq s(n) \succcurlyeq 1 / n
$$

it follows that there exists a polynomial $\mathbf{p}(x)$ such that

$$
\mathbf{S}^{\star}(x)+\mathbf{p}(x)+\log (1-x)
$$

has nonnegative coefficients. Then

$$
\mathbf{R}(x)=\exp \left(\mathbf{S}^{\star}(x)+\mathbf{p}(x)\right)
$$

has nonnegative coefficients. Since (6.2) has nonnegative coefficients it also follows that the exponential of (6.2) has nonnegative coefficients, that is,

$$
\left[x^{n}\right]((1-x) \cdot \mathbf{R}(x)) \geq 0 .
$$

Differentiating (6.3), and multiplying through by $x$, gives

$$
\begin{aligned}
x \mathbf{R}^{\prime}(x) & =x\left(\mathbf{S}^{\star^{\prime}}(x)+\mathbf{p}^{\prime}(x)\right) \cdot \mathbf{R}(x) \\
& =\left(x(1-x)^{-1}\left(\mathbf{S}^{\star^{\prime}}(x)+\mathbf{p}^{\prime}(x)\right)\right) \cdot(1-x) \mathbf{R}(x) .
\end{aligned}
$$

We will use Lemma 3.3 with

$$
\begin{aligned}
& \mathbf{A}(x)=x(1-x)^{-1}\left(\mathbf{S}^{\star \prime}(x)+\mathbf{p}^{\prime}(x)\right), \\
& \mathbf{B}(x)=(1-x) \mathbf{R}(x), \\
& \mathbf{C}(x)=x \mathbf{R}^{\prime}(x) .
\end{aligned}
$$

For $n$ larger than the degree of $\mathbf{p}(x)$, we have

$$
\begin{aligned}
a(n) & =\left[x^{n}\right]\left(x(1-x)^{-1} \mathbf{S}^{\star^{\prime}}(x)+x(1-x)^{-1} \mathbf{p}^{\prime}(x)\right) \\
& =s^{\star}(1)+\cdots+n s^{\star}(n)+\mathbf{p}^{\prime}(1) .
\end{aligned}
$$

From (6.1) we have $\mathbf{S}^{\star^{\prime}}(1)=\infty$, and thus

$$
\mathbf{p}^{\prime}(1)=\mathrm{o}\left(\sum_{m<n} m s^{\star}(m)\right) .
$$

From (6.5) and (6.6) it follows that

$$
a(n) \sim s^{\star}(1)+\cdots+n s^{\star}(n) .
$$

So by Lemma 5.3 and Proposition 2.4. $\mathbf{A}(x) \in \mathrm{RT}_{1}$. This is condition (a) of Lemma 3.3 .

$\mathbf{B}(x)$ has nonnegative coefficients by (6.4), and since $\mathbf{R}(x)$ also has nonnegative coefficients,

$$
0 \leq b(n)=\left[x^{n}\right](1-x) \mathbf{R}(x) \leq r(n)=\left[x^{n}\right] x \mathbf{R}^{\prime}(x) / n=c(n) / n ;
$$

so $b(n)=\mathrm{o}(c(n))$. This gives condition (b) of Lemma 3.3

From (6.4) we also have

$$
c(n)-c(n-1)=n r(n)-(n-1) r(n-1) \geq 0 .
$$

Hence condition (c) of Lemma 3.3 holds.

So, by Lemma 3.3. $\mathbf{C}(x) \in \mathrm{RT}_{1}$, that is, $x \mathbf{R}^{\prime}(x) \in \mathrm{RT}_{1}$. Then $\mathbf{R}(x) \in \mathrm{RT}_{1}$ by Proposition 2.4. So from Corollary 3.2 we have

$$
\mathbf{T}(x)=\exp (-\mathbf{p}(x)) \cdot \mathbf{R}(x) \in \mathbf{R T}_{1} .
$$


Finally, condition (c) implies $\mathbf{S}(1)=\infty$; so

$$
s(n)=\mathrm{o}\left(\left[x^{n}\right] \exp (\mathbf{S}(x))\right)
$$

by Lemma 4.4 Now $0 \leq s(n) \leq s^{\star}(n)$ leads to

$$
\left[x^{n}\right] \exp (\mathbf{S}(x)) \leq\left[x^{n}\right] \exp \left(\mathbf{S}^{\star}(x)\right)=\left[x^{n}\right] \mathbf{T}(x)
$$

and thus $s(n)=\mathrm{o}(t(n))$.

\section{COMPTON'S APPROACH TO LOGICAL LIMIT LAWS}

A class $\mathcal{K}$ of finite relational structures is said to be adequate if it is closed under disjoint union and components, and, up to isomorphism, it has only finitely many structures of each size. Let $p(n)$ count (up to isomorphism) the number of component structures in $\mathcal{K}$ of size $n$, and let $a(n)$ count the total number of structures in $\mathcal{K}$ of size $n$. The combinatorial identity connecting the two counting functions $a(n)$ and $p(n)$ is

$$
\mathbf{A}(x)=\exp \left(\mathbf{P}^{\star}(x)\right)
$$

where

$$
\begin{array}{ll}
\mathbf{A}(x)=\sum a(n) x^{n}, & \mathbf{P}^{\star}(x)=\sum p^{\star}(n) x^{n}, \\
p^{\star}(0)=0, & p^{\star}(n)=\sum_{j k=n} p(j) / k \text { for } n \geq 1 .
\end{array}
$$

The connection between adequate classes and (7.1) is very tight, for if $p(n)$ is any nonnegative integer-valued function with $p(0)=0$, then there is an adequate class $\mathcal{K}$ with $p(n)$ the count function for the components of $\mathcal{K}$, and the function $a(n)$ satisfying (7.1) is the total count function for $\mathcal{K}$.

Compton proved two main theorems for the purpose of finding classes $\mathcal{K}$ with logical limit laws. We assume that $\mathcal{K}$ is an adequate class of relational structures with the counting functions $a(n)$ and $p(n)$ as described above. Furthermore, we assume $a(n) \succ 02$ The striking feature of Compton's theorems is that he is able to prove logical limit laws just from knowing information about the counting function $a(n)$. Note that if $a(n) \in \mathrm{RT}_{\rho}$, then $0 \leq \rho \leq 1$ since the $a(n)$ are integers. We only consider $0<\rho \leq 1$ since Compton's method does not work for the case $\rho=0$. (The case $\rho=0$ requires more knowledge about $\mathcal{K}$ than just the count functions to determine if there is a logical limit law.)

Theorem 7.1 (Compton, 1987/1989). If $a(n) \in \mathrm{RT}_{1}$, then $\mathcal{K}$ has a monadic second-order zero-one law.

Theorem 7.2 (Compton, 1989). If $a(n) \in \mathrm{RT}_{\rho}$, where $0<\rho<1$, and if there exist $K$ and $C$ such that

$$
\frac{a(n-k)}{a(n)} \leq C \rho^{k} \quad \text { for } \quad K \leq k \leq n,
$$

then $\mathcal{K}$ has a monadic second-order limit law.

\footnotetext{
${ }^{2}$ This is the same as requiring that the gcd of the sizes of the components be 1.
} 


\section{A useful corollary}

For applications of Theorem 7.2 we use the following.

Corollary 8.1. Suppose $a(n) \in \mathrm{RT}_{\rho}$ and $\frac{a(n-1)}{a(n)} \preccurlyeq \rho$. Then $\mathcal{K}$ has a monadic second-order limit law.

Proof. Choose $K \geq 1$ such that

$$
n \geq K \Longrightarrow\left\{\begin{array}{l}
a(n)>0, \quad \text { and } \\
\frac{a(n-1)}{a(n)} \leq \rho .
\end{array}\right.
$$

Now suppose $K \leq k \leq n$.

Case 1: If $n-k \geq K$, then

$$
\frac{a(n-k)}{a(n)}=\frac{a(n-k)}{a(n-k+1)} \cdots \frac{a(n-1)}{a(n)} \leq \rho^{k} .
$$

Case 2: If $n-k<K$, then

$$
\begin{aligned}
\frac{a(n-k)}{a(n)} & =\frac{a(n-k)}{a(K)} \frac{a(K)}{a(n)} \\
& \leq \frac{a(n-k)}{a(K)} \rho^{n-K} \quad \text { by Case } 1 \\
& =\left(\frac{a(n-k)}{a(K)} \rho^{n-K-k}\right) \rho^{k} \\
& \leq\left(\frac{1}{a(K)} \cdot \max (a(0), \ldots, a(K-1)) \cdot \rho^{-K}\right) \rho^{k} .
\end{aligned}
$$

Thus Theorem 7.2 applies.

\section{Applications to logical limit laws}

Now we use our results to improve the scope of the conditions on $p(n)$ that lead to classes of structures covered by Compton's theorems.

Theorem 9.1. If $p(n) \in \mathrm{RT}_{1}$, then $\mathcal{K}$ has a monadic second-order zero-one law.

Proof. This follows from Theorems [6.2 and 7.1 after noting that $p(n)$ is always a nonnegative integer.

This theorem yields many classes of structures that, before, were not known to have a $0-1$ law. For example, if

$$
p(n) \sim a n^{b} e^{c n^{d}},
$$

with $d<1$ and $a, c>0$, then one has a monadic second-order $0-1$ law. Previously the known comprehensive general collection of count functions $p(n)$ that implied such a law was the family of polynomially bounded $p(n)$, that is, $p(n)=\mathrm{O}\left(n^{c}\right)$ for some $c$ (see Bell [1]). Now we have entire families of superpolynomial count functions $p(n)$ that guarantee such laws. 
Example 9.2. Let $\mathcal{K}$ be the class of finite directed graphs $\mathbf{G}=(G, R)$ satisfying the first-order conditions

$$
\begin{aligned}
& \forall x(\exists y(y R x) \leftrightarrow x R x), \\
& \forall x \forall y((x R x) \&(x R y) \rightarrow(y R x)), \\
& \forall x \forall y \forall z((x R x) \&(x R y) \&(y R z) \rightarrow(x R z)), \\
& \forall x(\neg(x R x) \rightarrow \exists ! y(x R y)) .
\end{aligned}
$$

Such a digraph consists of an equivalence relation plus a possibly empty collection of vertices that each have but one edge attached to them, and that edge is outgoing to an element in the equivalence relation. The vertices comprising the equivalence relation are precisely those in the range of the binary relation $R$. The indecomposable members of $\mathcal{K}$ are those with a single equivalence class. It is easy to see that $p(n)$ is the number of partitions of the integer $n$, which we know from the famous results of Hardy and Ramanujan to satisfy

$$
p(n) \sim \frac{1}{4 \sqrt{3}} n^{-1} e^{\sqrt{2 / 3} \pi n^{1 / 2}} .
$$

By Theorem 0.1 we see that $\mathcal{K}$ has a monadic second-order $0-1$ law.

Theorem 9.3. Suppose $0<\rho<1$. If

(a) $p(n) \in \mathrm{RT}_{\rho}$ and

(b) $\liminf _{n \rightarrow \infty} n p(n) \rho^{n}>1$,

then $\mathcal{K}$ has a monadic second-order limit law.

Proof. From Theorem6.1 we know $a(n) \in \mathrm{RT}_{\rho}$ as well as $\frac{a(n-1)}{a(n)} \prec \rho$; so Corollary 8.1 applies.

Prior to Theorem 9.3 all applications of Compton's Theorem 7.2 were based on the asymptotics of Knopfmacher, Knopfmacher, and Warlimont [11] that say if

$$
p(n)=C_{1} \beta^{n}+\mathrm{O}\left(\gamma^{n}\right), \quad 0<\gamma<\beta, \quad C_{1}>0,
$$

then

$$
a(n) \sim C_{2} \beta^{n} e^{2 \sqrt{a n}} / n^{3 / 4}, \quad \text { for some } C_{2}>0 .
$$

These results were proved by the well-known use of Cauchy's integral theorem for asymptotics. In this case,

$$
a(n)=\frac{1}{2 \pi i} \int_{\mathcal{C}} \exp \left(\mathbf{P}^{\star}(z)\right) \cdot \frac{d z}{z^{n+1}} .
$$

From the hypothesis (9.1) we immediately have $\rho=1 / \beta$ and $p(n) \in \mathrm{RT}_{\rho}$ as well as $\liminf _{n \rightarrow \infty} n p(n) \rho^{n}>1$. Thus Theorem 9.3 covers condition (9.1). But we can say much more. In the following corollary, we focus on generalizing the $C \beta^{n}$ asymptotics and drop any reference to an error term.

Corollary 9.4. For $\mu<1<\beta$ and $C>0$, or $\mu=1<\beta$ and $C>1$, the condition

$$
p(n) \sim C \beta^{n} / n^{\mu}
$$

guarantees that $\mathcal{K}$ has a monadic second-order limit law.

Proof. From Proposition $\left[2.4\right.$ we see that $p(n) \in \mathrm{RT}_{\rho}$, where $\rho=1 / \beta$, and the condition $\liminf _{n \rightarrow \infty} n p(n) \rho^{n}>1$ is readily verified. Thus Theorem 9.3 applies. 
Example 9.5. For a fixed positive integer $k$, let $\mathcal{K}$ be the class of finite $k$-colored linear forests. (The components of linear forests are chains. If $k=4$, then one can think of a member of $\mathcal{K}$ as a collection of DNA fragments.) Then

$$
p(n) \sim k^{n}
$$

so by Corollary 9.4 we know that $\mathcal{K}$ has a monadic second-order limit law.

Example 9.6. For $h$ a fixed positive integer, let $\mathcal{K}$ be the class of finite forests of planted plane trees of height at most $h$, that is, the class of finite graphs whose components are planted plane trees of height at most $h$.

The basic study of the generating function $\mathbf{P}(x):=\sum p(n) x^{n}$ for finite planted plane trees can be found in the 1972 paper [9] of de Bruijn, Knuth and Rice. From their work we know that

$$
\mathbf{P}(x)=\frac{f(x)}{g(x)}
$$

where $f(x)$ and $g(x)$ are the polynomials given by

$$
\begin{aligned}
& f(x)=2 x \cdot \frac{(1+\sqrt{1-4 x})^{h}-(1-\sqrt{1-4 x})^{h}}{\sqrt{1-4 x}}, \\
& g(x)=\frac{(1+\sqrt{1-4 x})^{h+1}-(1-\sqrt{1-4 x})^{h+1}}{\sqrt{1-4 x}} .
\end{aligned}
$$

They also note that the degree of $g(x)$ is $d=\lfloor h / 2\rfloor$, and that $g(x)$ has $d$ distinct positive roots $r_{j}$ given by

$$
r_{j}=\frac{1}{4} \sec ^{2}\left(\frac{j \pi}{h+1}\right) \quad \text { for } 1 \leq j \leq d .
$$

Of course we need $h \geq 2$ in order for $g(x)$ to have any roots. Clearly $0<r_{1}<\cdots<$ $r_{d}$, and for $h \geq 3$ the smallest root $r_{1}$ lies in the interval $(1 / 4,1 / 2]$.

Given that $h \geq 3$, for some $c \neq 0$ we have

$$
g(x)=c\left(x-r_{1}\right) \cdots\left(x-r_{d}\right) .
$$

So by the method of partial fractions,

$$
\frac{1}{g(x)}=\sum_{j=1}^{d} \frac{c_{j}}{x-r_{j}} \quad \text { where } c_{j}=c^{-1} \prod_{i \neq j}\left(r_{j}-r_{i}\right)^{-1} .
$$

From this we easily have

$$
\begin{aligned}
p(n) & =\left[x^{n}\right] \frac{f(x)}{g(x)} \\
& =-\sum_{j=1}^{d} c_{j} f\left(r_{j}\right) / r_{j}^{n+1} \quad \text { for } n \geq\left\lfloor\frac{h-1}{2}\right\rfloor(=\operatorname{deg}(f)) \\
& \sim-c_{1} \frac{f\left(r_{1}\right)}{r_{1}}\left(\frac{1}{r_{1}}\right)^{n},
\end{aligned}
$$

and thus for $h \geq 3$ the class $\mathcal{K}$ has a monadic second-order limit law by Corollary 9.4. In the cases $h=1,2$ the values $p(n)$ are uniformly bounded, and thus one has a monadic second-order 0-1 law. 
Remark 9.7. If we change the condition $\mu<1$ in Corollary 9.4 to $\mu>1$, then we can find examples of classes $\mathcal{K}$ with such a count function $p(n)$ that fail to have a first-order limit law.

Remark 9.8. We can construct, for any given $\rho$ with $0<\rho \leq 1$, an infinite sequence $\mathcal{K}_{m}$ of classes of finite relational structures with monadic second-order limit laws such that the count functions $p_{m}(n)$ and $a_{m}(n)$ for $\mathcal{K}_{m}$ are in $\mathrm{RT}_{\rho}$ and growing infinitely faster at each successive step; that is, we have

$$
p_{m}(n)=\mathrm{o}\left(a_{m}(n)\right) \text { and } a_{m}(n)=\mathrm{o}\left(p_{m+1}(n)\right) .
$$

To construct the sequence we start with

$$
p_{0}(n)=\left\lfloor 1 / \rho^{n}\right\rfloor, \quad \text { for } n \geq 1 .
$$

Then

$$
a_{0}(n)=\left[x^{n}\right] \exp \left(\mathbf{P}_{0}^{\star}(x)\right)
$$

gives $p_{0}(n)=\mathrm{o}\left(a_{0}(n)\right)$ by Theorem 6.1 or 6.2. Now observe that by modifying $a_{0}(n)$, by simply setting $a_{0}(0)$ to 0 , we have a sequence that can be used as a $p(n)$, satisfying the premises of Theorem 6.1 or 6.2. Inductively define $p_{k+1}$ and $a_{k+1}$ by: $p_{k+1}(0)=0$ and

$$
\begin{array}{lll}
p_{k+1}(n)=\left[x^{n}\right] \exp \left(\mathbf{A}_{k}^{\star}(x)\right), & & \text { for } n \geq 1, \\
a_{k+1}(n)=\left[x^{n}\right] \exp \left(\mathbf{P}_{k+1}^{\star}(x)\right), & & \text { for } n \geq 0,
\end{array}
$$

where $\mathbf{P}_{k}(x)=\sum_{n} p_{k}(n) x^{n}$ and $\mathbf{A}_{k}(x)=\sum_{n} a_{k}(n) x^{n}$. Now take classes $\mathcal{K}_{m}$ with counting functions $a_{m}(n)$ and $p_{m}(n)$.

In the case that $\rho=1$ these classes have, for $m \geq 1$, both counting functions with superpolynomial growth, growing far faster than the classes with polynomially bounded $p(n)$ that were, with minor exceptions, the only ones that were previously known to have a monadic second-order $0-1$ law. Likewise, with $0<\rho<1$, the classes $\mathcal{K}_{m}$, for $m \geq 1$, grow far faster than any examples that we knew before with a monadic second-order limit law.

\section{REFERENCES}

[1] Jason P. Bell, Sufficient conditions for zero-one laws. Trans. Amer. Math. Soc. 354 (2002), no. 2, 613-630. MR 2002j:60057

[2] Jason P. Bell, Edward A. Bender, Peter J. Cameron, and L. Bruce Richmond, Asymptotics for the probability of connectedness and the distribution of number of components. Electron. J. Combin. 7 (2000), R33. MR 2001d:05009

[3] Edward A. Bender, Asymptotic methods in enumeration. SIAM Rev. 16 (1974), 485-515. MR 51:12545

[4] Stanley N. Burris, Number Theoretic Density and Logical Limit Laws. Mathematical Surveys and Monographs, Vol. 86, Amer. Math. Soc., Providence, RI, 2001. MR 2002c:03060

[5] Stanley Burris, Spectrally determined first-order limit laws. Logic and Random Structures (New Brunswick, NJ, 1995), 33-52, ed. by Ravi Boppana and James Lynch. DIMACS Ser. Discrete Math. Theoret. Comput. Sci. 33, Amer. Math. Soc., Providence, RI, 1997. MR 98j:03047

[6] Stanley Burris and András Sárközy, Fine spectra and limit laws I. First-order laws. Canad. J. Math. 49 (1997), 468-498. MR 98k:03076a

[7] Kevin J. Compton, A logical approach to asymptotic combinatorics. I. First order properties. Adv. in Math. 65 (1987), 65-96. MR 88k:03065

[8] Kevin J. Compton, A logical approach to asymptotic combinatorics. II. Monadic second-order properties. J. Combin. Theory, Ser. A 50 (1989), 110-131. MR 90c:03024

[9] N. G. de Bruijn, D. E. Knuth, and S. O. Rice, The average height of planted plane trees. Graph Theory and Computing, Academic Press, New York, 1972, pp. 15-22. MR 58:21737 
[10] R. Durrett, B. Granovsky, and S. Gueron, The equilibrium behavior of reversible coagulationfragmentation processes. J. Theoret. Probab. 12 (1999), 447-474. MR 2000g:82013

[11] Arnold Knopfmacher, John Knopfmacher, and Richard Warlimont, "Factorisatio numerorum" in arithmetical semigroups. Acta Arith. 61 (1992), 327-336. MR 93f:11069

Mathematics Department, University of Michigan, East Hall, 525 East University, Ann Arbor, Michigan 48109-1109

E-mail address: belljp@umich.edu

Department of Pure Mathematics, University of Waterloo, Waterloo, Ontario N2L 3G1 CANADA

E-mail address: snburris@thoralf.uwaterloo.ca

$U R L$ : www.thoralf . uwaterloo.ca 